Pacific Journal of Mathematics

A GENERALIZATION OF A THEOREM OF ATKINSON TO 


\section{A GENERALIZATION OF A THEOREM OF ATKINSON TO NON-INVARIANT MEASURES}

\section{Daniel UllmaN}

We prove that, if $T$ is an ergodic, conservative, non-singular automorphism of a Lebesgue space $(X, \mu)$, then the following are equivalent for $f$ in $L^{1}(\mu)$ :

(1) If $\mu(B)>0$ and $\varepsilon>0$, then there is an integer $n \neq 0$ such that

$$
\mu\left(B \cap T^{-n} B \cap\left\{x:\left|\sum_{j=0}^{n-1} f\left(T^{j} x\right) \cdot \frac{d \mu \circ T^{j}}{d \mu}(x)\right|<\varepsilon\right\}\right)>0 .
$$

(2) $\liminf _{n \rightarrow \infty}\left|\sum_{j=0}^{n-1} f\left(T^{j} x\right) \cdot \frac{d \mu \circ T^{j}}{d \mu}(x)\right|=0 \quad$ for a.e. $x$.

(3) $\int f d \mu=0$.

Our basic objects of study are a non-atomic Lebesgue space $(X, \mathscr{B}, \mu)$ and a conservative, aperiodic, non-singular automorphism $T: X \rightarrow X$. Associated with any measurable function $f: X \rightarrow \mathbf{R}^{n}$ is a cocycle $f^{*}: \mathbf{Z} \times$ $X \rightarrow \mathbf{R}^{n}$ defined by

$$
f^{*}(n, x)= \begin{cases}\sum_{k=0}^{n-1} f\left(T^{k} x\right), & n>0 \\ 0, & n=0 \\ -f^{*}\left(-n, T^{n} x\right), & n<0\end{cases}
$$

$f^{*}$ satisfies the so-called cocycle identity:

$$
f^{*}(m+n, x)=f^{*}(m, x)+f^{*}\left(n, T^{m} x\right),
$$

for all integers $m$ and $n$ and for a.e. $x \in X$.

The non-singularity of $T$ permits us to define the Radon-Nikodym derivative

$$
\omega_{k}(x)=\frac{d \mu \circ T^{k}}{d \mu}(x) \text { for } k \in \mathbf{Z} \text {, a.e. } x \in X
$$


We can use this to build what we call an $H$-cocycle-after Halmos [4], Hopf [5], and Hurewicz [6] - defined by

$$
f_{*}(n, x)= \begin{cases}\sum_{m=0}^{n-1} \omega_{m}(x) f\left(T^{m} x\right) & \text { if } n>0 \\ 0 & \text { if } n=0 \\ -\omega_{n}(x) f_{*}\left(-n, T^{n} x\right) & \text { if } n<0\end{cases}
$$

The quotient ergodic theorem [3] asserts that, for an integrable $f$, the rate of growth of $f_{*}(n, x)$ depends only on the integral $\int f d \mu$. Analogous to (1) is the $H$-cocycle identity:

$$
f_{*}(m+n, x)=f_{*}(m, x)+\omega_{m}(x) f_{*}\left(n, T^{m} x\right) .
$$

When $T$ is measure-preserving, the $H$-cocycle coincides with the usual cocycle.

Suppose $B \in \mathscr{B}$. A cocycle or an $H$-cocycle $f(n, x)$ is recurrent on $B$ if, for all $\varepsilon>0$,

$$
\mu\left(\bigcup_{n \neq 0} B \cap T^{-n} B \cap\{x \in X \ni|f(n, x)|<\varepsilon\}\right)>0 .
$$

A cocycle or an $H$-cocycle $f(n, x)$ is recurrent if it is recurrent on all sets of positive measure. We call a function $f: X \rightarrow \mathbf{R}^{n}$ recurrent if $f^{*}(n, x)$ is, and we call it $H$-recurrent if $f_{*}(n, x)$ is.

These definitions coincide with the classical notion of recurrence (or sometimes "persistence") of random walks, introduced by Polya [8], who proved that the Bernoulli random walk on $\mathbf{Z}^{n}$ is recurrent (that is, bound to return to zero) if and only if $n=1$ or 2. Later, Chung and Fuchs [2] proved that a random walk on $\mathbf{R}$ based on an increment random variable $X$ of finite mean is recurrent if and only if $E X=0$. In 1976, Atkinson [1] discovered the following beautiful result, extending the theorem of Chung and Fuchs to random walks with non-independent increments.

THEOREM (ATKINSON). If $T$ is ergodic and preserves a finite measure $\mu$ and $f$ is a real, integrable function on $X$, then $f$ is recurrent if and only if $\int f d \mu=0$.

The following result further extends the theorem of Chung and Fuchs to the non-stationary case.

THEOREM. If $T$ is an ergodic, conservative, non-singular automorphism of a Lebesgue space $(X, \mathscr{B}, \mu)$ and if $f: X \rightarrow \mathbf{R}$ is integrable, then the following conditions are equivalent:

(1) $f_{*}$ is H-recurrent, 
(2) $\liminf \left|f_{*}(n, x)\right|=0$ for a.e. $x \in X$, and

(3) $\int f d \mu=0$.

Proof. The first thing to notice is that once we know this theorem for a measure $\mu$, we know it for all measures $\nu$ equivalent to $\mu$. To see this, note that the $H$-cocycle $f_{*}$ built from $f$ under $(X, \mathscr{B}, \nu, T)$ is related to the $H$-cocycle $f^{\prime}{ }_{*}$ built from $f^{\prime}=f \cdot d \nu / d \mu$ under $(X, \mathscr{B}, \mu, T)$ by the equation

$$
f_{*}^{\prime}(n, x)=\frac{d \nu}{d \mu}(x) \cdot f_{*}(n, x)
$$

This shows that $f^{\prime}$ * gets small exactly when $f_{*}$ gets small. Since $\int f d \nu=0$ exactly when $\int f^{\prime} d \mu=0$, we inherit the result for $f$ and $\nu$ from the result for $f^{\prime}$ and $\mu$.

In particular, since this theorem reduces to Atkinson's theorem if $T$ preserves $\mu$, we have the result for any dynamical system $(X, \mathscr{B}, \mu, T)$ with an equivalent finite invariant measure. We also see that there is no loss of generality in assuming that $\mu X=1$ and we proceed under this assumption.

(1) $\Rightarrow$ (2) Let $D=\left\{x \in X \ni \liminf \left|f_{*}(n, x)\right|>\varepsilon\right\}$ for some $\varepsilon>0$. If $\mu D>0$, then there would be an integer $N$ so large that

$$
C=\left\{x \in D \ni\left|f_{*}(n, x)\right|>\varepsilon \text { for all } n \text { with }|n|>N\right\}
$$

would have positive measure. One could then find a set $B \subset C$ of positive measure disjoint from its first $N$ forward and backward translates. (Just remove from $C$ points that return too soon under $T$ or $T^{-1}$ and use Kac's recurrence theorem [7].) Then

$$
\mu\left(B \cap T^{-n} B \cap\left\{x \ni\left|f_{*}(n, x)\right|<\varepsilon\right\}\right)=0
$$

for all integers $n \neq 0$, which contradicts the $H$-recurrence of $f$.

$(2) \Rightarrow(3)$ This implication is proved via a simple application of the quotient ergodic theorem [3]. Let $g$ be the constant function 1. Since $g_{*}(n, x)>1$ for every $x$ and all positive $n$,

$$
\left|f_{*}(n, x)\right| \geq\left|\frac{f_{*}(n, x)}{g_{*}(n, x)}\right| \stackrel{\text { a.e. }}{\rightarrow} \frac{\left|\int f d \mu\right|}{\left|\int g d \mu\right|}=\left|\int f d \mu\right| .
$$

If $\int f d \mu \neq 0$, this last quantity is positive and so $\liminf \left|f_{*}(n, x)\right|>0$ for a.e. $x \in X$. 
(3) $\Rightarrow(1)$ This argument encompasses the remainder of the paper. Three important estimates are isolated as lemmas.

Assume $f_{*}$ is transient-i.e., not recurrent. This means that there is a set $B \in \mathscr{B}$ with $\mu B>0$ and a $\delta>0$ such that

$$
\mu\left(B \cap T^{-n} B \cap\left\{x \ni\left|f_{*}(n, x)\right|<\delta\right\}\right)=0 \quad \forall n \neq 0 .
$$

Let $A$ be a subset of $B$ with $\mu A=\mu B$ and such that

$$
A \cap T^{-n} A \cap\left\{x \ni\left|f_{*}(n, x)\right|<\delta\right\}=\varnothing \text { for all } n \neq 0 .
$$

By $\chi$ we will mean $\chi_{A}$, the characteristic function of the set $A$.

For all $\varepsilon>0$ and a.e. $x$, the quotient ergodic theorem tells us that

$$
\left|\frac{\chi_{*}(n, x)}{g_{*}(n, x)}-\mu A\right|<\varepsilon \text { for sufficiently large } n \text {. }
$$

Another way to write this is to define the "weight" $w(j, x)$ of the integer $j$, depending on $x$, by:

$$
w(j, x)= \begin{cases}\omega_{j}(x) & \text { if } T^{j} x \in A, \\ 0 & \text { otherwise. }\end{cases}
$$

For the remainder of the proof, fix $x$ such that (5) holds (for an $\varepsilon$ to be specified later) and such that $f_{*}(n, x) / g_{*}(n, x) \rightarrow \int f d \mu$. Then (5) translates to

$$
\left|\sum_{j=0}^{n-1} w(j, x)-\mu A \cdot g_{*}(n, x)\right|<\varepsilon \cdot g_{*}(n, x) .
$$

We call an integer $j$ good if $T^{j} x \in A$. Note that the previous summation has non-zero contribution only from good indices $j$. For good $m$, let $I_{m}$ be the interval on the real line centered at $f_{*}(m, x)$ and of radius (i.e., half-length) equal to $w(m, x) \delta$. Let $\lambda$ be Lebesgue measure on the line.

LEMMA 1. If $m$ is good, $f_{*}(j, x) \in I_{m}$ only when $j=m$.

Proof of Lemma. That $m$ is good means that $T^{m} x \in A$, which implies that

$$
\left|f_{*}\left(j-m, T^{m} x\right)\right| \geq \delta \text { for any } j \neq m .
$$

The $H$-cocycle identity (2) can be written

$$
f_{*}\left(j-m, T^{m} x\right)=\frac{f_{*}(j, x)-f_{*}(m, x)}{w(m, x)} .
$$

Hence equation (7) implies that $\left|f_{*}(j, x)-f_{*}(m, x)\right|>w(m, x) \delta$, which is what it means to say that $f_{*}(j, x) \notin I_{m}$. 
The intervals $I_{m}$ may be of widely varying size. Yet the following lemma assures us that no $I_{m}$ for large $m$ can be nearly as long as the sum of lengths of $I_{j}$ for $0 \leq j<m$.

LEMMA 2. If $m$ is good and sufficiently large, then

$$
w(m, x)<\frac{1}{10} \sum_{j=0}^{m-1} w(j, x) .
$$

Proof of Lemma. Choose $n$ large enough so that equation (6) holds for all $m>n$. Write

$$
w(m, x)=\sum_{j=0}^{m} w(j, x)-\sum_{j=0}^{m-1} w(j, x)
$$

and

$$
\mu A \cdot w(m, x)=\mu A \cdot g_{*}(m+1, x)-\mu A \cdot g_{*}(m, x) .
$$

Subtracting the last equation from the one before yields

$$
\begin{aligned}
w(m, x)[1-\mu A] & \leq \varepsilon g_{*}(m+1, x)+\varepsilon g_{*}(m, x) \\
& =2 \varepsilon g_{*}(m, x)+\varepsilon w(m, x)
\end{aligned}
$$

if $m>n$, using (6).

Rearranging:

$$
w(m, x)[1-\mu A-\varepsilon] \leq 2 \varepsilon g_{*}(m, x) .
$$

If $\varepsilon$ is sufficiently small, the quantity in square brackets is positive, and so we get

$$
\begin{aligned}
w(m, x) & \leq \frac{2 \varepsilon}{(1-\mu A-\varepsilon)} g_{*}(m, x) \\
& \leq\left[\frac{2 \varepsilon}{(\mu A-\varepsilon)(1-\mu A-\varepsilon)}\right] \sum_{j=0}^{m-1} w(j, x)
\end{aligned}
$$

where the second inequality comes from (6). Simply choose $\varepsilon$ small enough so that the quantity in (8) in square brackets is less than $1 / 10$ and the lemma is proved.

Let $J_{n}$ be the convex hull of $\left\{f_{*}(j, x) \ni 0 \leq j<n\right\} . J_{n}$ is the shortest interval on the real line containing the first $n f_{*}(j, x)$ 's. Our goal now is to show that the intervals $J_{n}$ have bounded weight density. 
LEMMA 3. For sufficiently large $n$

$$
\sum_{j=0}^{n-1} w(j, x)<\frac{4}{\delta} \lambda J_{n}
$$

Proof of Lemma. Let $\mathfrak{\Im}=\left\{I_{m} \ni m\right.$ is good and $\left.0 \leq m<n\right\}$. $\mathfrak{\Im}$ is a collection of possibly overlapping intervals of varying sizes. Let $\mathfrak{\mho}^{\prime}$ be a subset of $\mathfrak{s}$ whose union equals that of $\mathfrak{s}$ and which is minimal with respect to this property. Call $m$ select if $I_{m} \in \mathfrak{\Im}^{\prime}$. Then

$$
\begin{aligned}
4 \lambda J_{n} & >2 \lambda\left(\bigcup_{m \text { select }} I_{m}\right)>\sum_{m \text { select }} \lambda I_{m} \\
& >\sum_{m \text { select }} \sum_{j \ni f_{*}(n, x) \in I_{m}} \delta w(j, x)>\delta \sum_{j=0}^{n-1} w(j, x) .
\end{aligned}
$$

The first inequality comes from Lemma 2 . The second inequality holds because the choice of $\mathfrak{J}^{\prime}$ forces all real numbers to lie in at most two $I_{m}$ with select indices $m$. The third inequality is just Lemma 1, and the fourth expresses the fact that every $f_{*}(j, x)$ with $0 \leq j<n$ and $j$ good lies in some select $I_{m}$. The lemma is proved.

It is now a simple matter to complete the proof of the theorem. Equation (6) says that, for all $\varepsilon>0$,

$$
\sum_{j=0}^{n-1} w(j, x)>g_{*}(n, x)(\mu A-\varepsilon),
$$

if $n$ is large enough. Hence Lemma 3 tells us that

$$
\lambda J_{n}>\frac{\delta}{4} g_{*}(n, x)(\mu A-\varepsilon) .
$$

This implies that

$$
\sup _{0 \leq j<n}\left|f_{*}(j, x)\right|>\frac{\delta}{8}(\mu A-\varepsilon) g_{*}(n, x) .
$$

Thus, for infinitely many $n$,

$$
\frac{\left|f_{*}(n, x)\right|}{g_{*}(n, x)}>\frac{\delta}{8}(\mu A-\varepsilon)>0,
$$

if $\varepsilon$ is small enough.

But the left-hand-side of this expression approaches $\left|\int f d \mu\right|$, which is seen to be, as required, greater than zero. 
I would like to thank Jack Feldman for suggesting this problem and for many helpful discussions.

\section{REFERENCES}

[1] G. Atkinson, Recurrence of co-cycles and random walks, J. London Math. Soc., (2) 13 (1976), 486-488.

[2] K. L. Chung, and W. Fuchs, On the distribution of values of sums of random variables, Mem. Amer. Math. Soc., 6 (1951), 1-12.

[3] R. V. Chacon and D. S. Ornstein, A general ergodic theorem, Illinois J. Math., 4 (1960), 153-160.

[4] P. R. Halmos, An ergodic theorem, Proc. Nat. Acad. Sci., 32 (1946), 156-161.

[5] E. Hopf, Ergodentheorie, Ergebnisse der Math., Berlin, 1937.

[6] W. Hurewicz, Ergodic theory without invariant measure, Annals of Math., 45 (1944), 192-206.

[7] M. Kacz, On the notion of recurrence in discrete stochastic processes, Annals of Math. Stat., 53 (1947), 1002-1010.

[8] G. Polya, Eine Ergänzung zu dem Bernoullischen Satz der Wahrscheinlichkeitsrechnung, Nach. Ges. Will. Gottingen, (1921), 223-228.

Received May 3, 1986 and in revised form July 15, 1986. This research was supported in part by NSF grant DMS84-03182 and constituted part of the authors Ph.D. dissertation.

The George Washington University

WASHINGTON, DC 20052 



\section{PACIFIC JOURNAL OF MATHEMATICS EDITORS}

V. S. VARADARAJAN (Managing Editor) University of California Los Angeles, CA 90024 HERBERT ClEMENS University of Utah Salt Lake City, UT 84112

R. FINN

Stanford University

Stanford, CA 94305

\section{HERMANN FLASCHKA} University of Arizona Tucson, AZ 85721

RAMEsh A. GANGolli University of Washington Seattle, WA 98195

VAUghan F. R. JONES

University of California Berkeley, CA 94720

\section{ROBION KIRBY}

University of California

Berkeley, CA 94720

C. C. MOORE

University of California

Berkeley, CA 94720

HAROLD STARK

University of California, San Diego

La Jolla, CA 92093

\section{ASSOCIATE EDITORS}
R. ARENS
E. F. BECKENBACH
B. H. NEUMANN
F. WOLF
K. YOSHIDA (1906-1982)

\section{SUPPORTING INSTITUTIONS}

UNIVERSITY OF ARIZONA

UNIVERSITY OF BRITISH COLUMBIA

CALIFORNIA INSTITUTE OF TECHNOLOGY

UNIVERSITY OF CALIFORNIA

MONTANA STATE UNIVERSITY

UNIVERSITY OF NEVADA, RENO

NEW MEXICO STATE UNIVERSITY

OREGON STATE UNIVERSITY
UNIVERSITY OF OREGON

UNIVERSITY OF SOUTHERN CALIFORNIA

STANFORD UNIVERSITY

UNIVERSITY OF HAWAII

UNIVERSITY OF TOKYO

UNIVERSITY OF UTAH

WASHINGTON STATE UNIVERSITY

UNIVERSITY OF WASHINGTON 


\section{Pacific Journal of Mathematics}

Vol. 130, No. $1 \quad$ September, 1987

K. Adachi, Continuation of bounded holomorphic functions from certain subvarieties to weakly pseudoconvex domains $\ldots \ldots \ldots \ldots \ldots \ldots \ldots$

Erazm Jerzy Behr, Enveloping algebras of Lie superalgebras ........... 9

Dong M. Chung, Scale-invariant measurability in abstract Wiener spaces . . . 27

Peter Gerard Dodds and Bernardus de Pagter, Algebras of unbounded

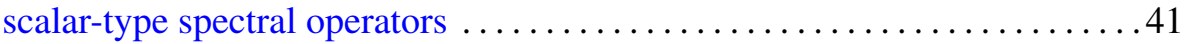

Wu-Yi Hsiang and Hsueh-Ling Huynh, Generalized rotational

hypersurfaces of constant mean curvature in the Euclidean spaces. II . . . .75

Harvey Bayard Keynes and M. Sears, Time changes for $\mathbf{R}^{n}$ flows and suspensions ..................................... 97

Frances Kirwan, Ronnie Lee and Steven Howard Weintraub, Quotients

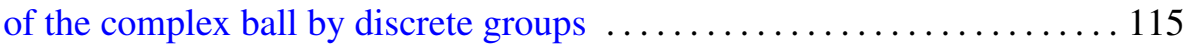

Magnhild Lien, Groups of knots in homology 3-spheres that are not

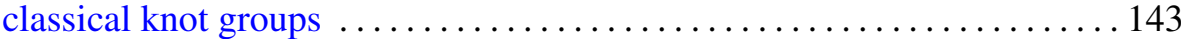

Juan Carlos Migliore, Liaison of a union of skew lines in $\mathbf{P}^{4} \ldots \ldots \ldots \ldots 153$

Jesper M. Møller, Spaces of sections of Eilenberg-Mac Lane fibrations . . . . 171 Daniel Ullman, A generalization of a theorem of Atkinson to noninvariant

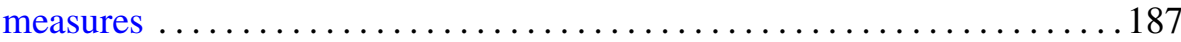

Kohhei Yamaguchi, Operations which detect $\mathscr{P}^{1}$ in odd primary connective

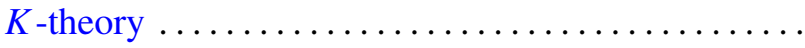

